\title{
DYNAMICS OF A DELAYED POPULATION MODEL WITH FEEDBACK CONTROL
}

\author{
WANG WENDI ${ }^{1}$ and TANG CHUNLEI ${ }^{1}$
}

(Received 14 July 1995)

\begin{abstract}
This paper studies a system proposed by $\mathrm{K}$. Gopalsamy and $\mathrm{P}$. X. Weng to model a population growth with feedback control and time delays. Sufficient conditions are established under which the positive equilibrium of the system is globally attracting. The conjecture proposed by Gopalsamy and Weng is here confirmed and improved.
\end{abstract}

\section{Introduction}

In [3], Gopalsamy and Weng proposed a system to model a population growth with time delay and feedback control:

$$
\begin{aligned}
& n^{\prime}(t)=r n(t)[1-n(t-\tau) / k-c u(t)], \\
& u^{\prime}(t)=-a u(t)+b n(t-\tau),
\end{aligned}
$$

where $r, k, a, b, c$ and $\tau$ are positive constants, $n(t)$ represents the population density at time $t$ and $u$ denotes an "indirect control" variable. One can see that system (1) has a positive equilibrium $\left(n^{*}, u^{*}\right)$ where

$$
n^{*}=\frac{a k}{a+k b c} \text { and } \quad u^{*}=\frac{b k}{a+k b c} .
$$

Gopalsamy and Weng also proved that the condition

$$
k\left(\frac{b c}{a}+\frac{r \tau}{n^{*}}\right) \exp (r \tau)<\frac{1}{2}
$$

ensures that any positive solution $(n(t), u(t))$ of system (1) satisfies

$$
\lim _{t \rightarrow+\infty}(n(t), u(t))=\left(n^{*}, u^{*}\right)
$$

\footnotetext{
'Department of Mathematics, Southwest Normal University, Chongqing, 400715, P. R. China. (C) Australian Mathematical Society 2000, Serial-fee code 0334-2700/00
} 
They conjectured that (4) remains valid if condition (3) is relaxed to

$$
k\left(\frac{b c}{a}+\frac{r \tau}{n^{*}}\right) \exp (r \tau)<1
$$

or to the even weaker condition

$$
k\left(\frac{b c}{a}+\frac{r \tau}{n^{*}}\right)<1
$$

In [4], Wang and Ma showed that condition (5) is sufficient for (4) to hold. Therefore, the first part of the conjecture is confirmed. We now come to the key result of this paper.

THEOREM 1. Each positive solution of system (1) satisfies

$$
\lim _{t \rightarrow+\infty}(n(t), u(t))=\left(n^{*}, u^{*}\right)
$$

provided that

$$
k b c / a+r \tau<1
$$

Since $n^{*}=k a /(a+k b c)$, we see that $n^{*}<k$, and therefore that condition (7) is weaker than (6). This means that the conjecture proposed by Gopalsamy and Weng is completely confirmed and improved.

\section{Proof of the main result}

Let $R_{+}^{2}=\{(n, u): n \geq 0 ; u \geq 0\}$ and let $C\left([-\tau, 0], R_{+}^{2}\right)$ denote the space of continuous functions mapping the interval $[-\tau, 0]$ into $R_{+}^{2}$. For ecological reasons, we shall assume that the initial conditions of system (1) take the form:

$$
\begin{gathered}
n(s)=\varphi_{1}(s), \quad s \in[-\tau, 0] ; \varphi_{1}(0)>0, \\
u(s)=\varphi_{2}(s), \quad s \in[-\tau, 0] ; \varphi_{2}(0)>0, \\
\left(\varphi_{1}, \varphi_{2}\right) \in C\left([-\tau, 0], R_{+}^{2}\right) .
\end{gathered}
$$

One can prove by the method of steps that any solution $(n(t), u(t))$ of system (1) corresponding to initial condition (8) is defined for all $t \geq 0$ and satisfies $n(t)>0$ and $u(t)>0$ for all $t>0$. Such solutions are called positive solutions.

A function $x(t)$ defined on $[0,+\infty)$ is said to be oscillatory about $x^{*}$ if there exists a sequence $\left\{t_{n}\right\}, t_{n} \rightarrow \infty$ as $n \rightarrow \infty$, such that $x\left(t_{n}\right)=x^{*}, n=1,2, \ldots$ Otherwise, we call $x(t)$ nonoscillatory about $x^{*}$. 
LEMMA 1. Let (7) hold. Then $k \exp (r \tau)<a /(b c)$.

Proof. By (7), we have

$$
k \exp (r \tau)<a(1-r \tau) \exp (r \tau) /(b c)
$$

Hence it suffices to show $(1-r \tau) \exp (r \tau)<1$. Let $\alpha(t)=(1-t) e^{t}-1$. Then $\alpha(0)=0$ and $\alpha^{\prime}(t)=-t e^{t}<0$ when $t>0$. As a consequence, $\alpha(t) \leq 0$ for $t \geq 0$. Lemma 1 now follows.

Let $M_{0}=k \exp (r \tau)$. Then Lemma 1 implies that one can choose $\epsilon>0$ such that

$$
L_{0} \triangleq k\left(1-\epsilon-b c M_{0} / a\right) \exp \left\{\left(1-\epsilon-M_{0} / n^{*}\right) r \tau\right\}>0 .
$$

We further restrict $\epsilon$ so that $a \epsilon / 2<b c M_{0}$.

Let sequences $\left\{M_{n}\right\}$ and $\left\{L_{n}\right\}$ be defined by

$$
\begin{array}{ll}
M_{i}=k\left(1+\frac{\epsilon}{i+1}-\frac{b c L_{i-1}}{a}\right) \exp \left\{\left(1+\frac{\epsilon}{i+1}-\frac{L_{i-1}}{n^{*}}\right) r \tau\right\}, \quad i=1,2 \ldots, \\
L_{i}=k\left(1-\frac{\epsilon}{i+1}-\frac{b c M_{i}}{a}\right) \exp \left\{\left(1-\frac{\epsilon}{i+1}-\frac{M_{i}}{n^{*}}\right) r \tau\right\}, \quad i=1,2 \ldots
\end{array}
$$

LEMMA 2. Let (7) hold. Then for any positive solution $(n(t), u(t))$ of system (1), there exist sequences $\left\{t_{i}\right\}$ and $\left\{t_{i}^{*}\right\}, t_{i} \rightarrow+\infty$ and $t_{i}^{*} \rightarrow+\infty$ as $i \rightarrow \infty$, such that

$$
\begin{array}{ll}
n(t)<M_{i} & \text { for } t>t_{i}, \\
n(t)>L_{i} & \text { for } t>t_{i}^{*} .
\end{array}
$$

PROOF. We first verify the case where $i=0$. There are two possibilities to be considered: $n(t)$ is nonoscillatory about $k$ or is oscillatory about $k$.

In the first case, there exists a $T_{0}>0$ such that $n(t)>k$ for all $t>T_{0}$ or $n(t)<k$ for all $t>T_{0}$. If the second alternative occurs, it is evident that $n(t)<M_{0}$ for all $t>T_{0}$. Suppose that $n(t)>k$ for all $t>T_{0}$. Then $n^{\prime}(t)<0$ for all $t>T_{0}+\tau$. It follows that $n(t)$ is decreasing when $t>T_{0}+\tau$, and therefore, there exists a constant $A$ such that $n(t) \rightarrow A$ as $t \rightarrow+\infty$. Moreover, one can easily show that $A \leq k$. Note that $k<M_{0}$. We may conclude that there exists a $T_{1}>T_{0}+\tau$ such that $n(t)<M_{0}$ for all $t>T_{1}$.

Let us now suppose that $n(t)$ is oscillatory about $k$ and let $n(\bar{t})$ denote an arbitrary local maximum of $n(t)$. Then we have

$$
0=n^{\prime}(\bar{t})<r n(\bar{t})(1-n(\bar{t}-\tau) / k)
$$


and therefore

$$
n(\bar{t}-\tau)<k
$$

It is easy to see that

$$
n^{\prime}(t) / n(t)<r
$$

Integrating (13) from $\bar{t}-\tau$ to $\bar{t}$ and using (12), we obtain

$$
n(\bar{t})<k \exp (r \tau)=M_{0}
$$

Since $n(\bar{t})$ is an arbitrary local maximum of $n(t)$, one can conclude that there exists a $T_{2}>0$ such that $n(t)<M_{0}$ for all $t>T_{2}$.

Let $t_{0}=\max \left\{T_{1}, T_{2}\right\}$. The above discussions indicate that $n(t)<M_{0}$ for all $t>t_{0}$. We show below that there exists a $t_{0}^{*}$ such that $n(t)>L_{0}$ for all $t>t_{0}^{*}$. By the second equation of system (1), one obtains

$$
u^{\prime}(t)<-a u(t)+b M_{0} \text { for } t>t_{0} .
$$

It follows that there exists $T_{3}>t_{0}$ such that

$$
u(t)<b M_{0} / a+\epsilon / c \text { for } t>T_{3},
$$

in which $\epsilon>0$ is the same as before. As a consequence, we have

$$
n^{\prime}(t)>r n(t)\left[1-n(t-\tau) / k-\left(b c M_{0} / a+\epsilon\right)\right]
$$

for $t>T_{3}$. Using arguments similar to those above, one can show from (15) that there exists a $t_{0}^{*}>T_{3}+\tau$ such that for $t>t_{0}^{*}$,

$$
n(t)>k\left(1-\epsilon-\frac{b c M_{0}}{a}\right) \exp \left\{\left(1-\epsilon-\frac{M_{0}}{n^{*}}\right) r \tau\right\}=L_{0},
$$

in which the equality $b c / a+1 / k=1 / n^{*}$ is used. This verifies the conclusion that Lemma 2 holds for $i=0$.

Now suppose that the lemma is true for $i$. Then there exists a $t_{i}^{*}$ such that $n(t)>L_{i}$ for $t>t_{i}^{*}$. It follows that there exists a $T_{4}>t_{i}^{*}$ such that

$$
u(t)>\frac{b L_{i}}{a}-\frac{\epsilon}{(i+2) c} \text { for } t>T_{4}
$$

which yields

$$
n^{\prime}(t)<r n(t)\left[1+\epsilon /(i+2)-b c L_{i} / a-n(t-\tau) / k\right] \text { for } t>T_{4} .
$$


By an argument similar to those used above, we may show that there exists a $t_{i+1}$ such that

$$
n(t)<k\left(1+\frac{\epsilon}{i+2}-\frac{b c L_{i}}{a}\right) \exp \left\{\left(1+\frac{\epsilon}{i+2}-\frac{L_{i}}{n^{*}}\right) r \tau\right\}=M_{i+1}
$$

for $t>t_{i+1}$. By this one can also show that there exists a $t_{i+1}^{*}$ such that

$$
n(t)>k\left(1-\frac{\epsilon}{i+2}-\frac{b c M_{i+1}}{a}\right) \exp \left\{\left(1-\frac{\epsilon}{i+2}-\frac{M_{i+1}}{n^{*}}\right) r \tau\right\}=L_{i+1}
$$

for $t>t_{i+1}^{*}$. Thus the induction is complete and the lemma is proved.

LEMMA 3. Under the assumptions of Lemma $2, M_{i}$ is monotonically increasing and $L_{i}<M_{i}$.

We omit the proof of this lemma since it is similar to that of Gopalsamy (see [2]).

Set $m=\lim _{i \rightarrow \infty} M_{i}$ and $l=\lim _{i \rightarrow \infty} L_{i}$. It is easy to see that

$$
\begin{aligned}
m & =k(1-b c l / a) \exp \left\{\left(1-l / n^{*}\right) r \tau\right\}, \\
l & =k(1-b c m / a) \exp \left\{\left(1-m / n^{*}\right) r \tau\right\}
\end{aligned}
$$

Put

$$
f(x)=k(1-b c x / a) \exp \left\{\left(1-x / n^{*}\right) r \tau\right\}
$$

and denote $f(f(x))$ by $f^{2}(x)$. Then (16) and (17) imply that $m$ and $l$ are the fixed points of $f^{2}(x)$. It is easy to see that $n^{*}$ is also a fixed point of $f^{2}(x)$. To establish $m=l$, we therefore need only to make the fixed points of $f^{2}(x)$ unique.

LEMMA 4. Let (7) hold. Then $f^{2}(x)$ has a unique fixed point in $[0, a /(b c)]$.

PROOF. We verify that $f^{2}(x)$ satisfies the conditions of the contraction mapping principle. By direct calculation, one obtains

$$
f^{\prime}(x)=k\left(-\frac{b c}{a}-\frac{r \tau}{n^{*}}+\frac{r \tau b c x}{a n^{*}}\right) \exp \left\{\left(1-x / n^{*}\right) r \tau\right\},
$$

which implies that $f(x)$ is strictly decreasing on $[0, a /(b c)]$. Observe that $f(0)=$ $k \exp (r \tau)<a /(b c)$ and $f(a /(b c))=0$. We know $f([0, a /(b c)]) \subset[0, a /(b c)]$. As a consequence, we have $f^{2}([0, a /(b c)]) \subset[0, a /(b c)]$. 
We show below that $f^{2}$ is contracting on $[0, a /(b c)]$. Set $g(x)=f^{2}(x)=f(f(x))$. Then we have

$$
\begin{aligned}
g^{\prime}(x)= & f^{\prime}[f(x)] f^{\prime}(x) \\
= & k^{2}\left[\frac{b c}{a}+\frac{r \tau}{n^{*}}-\frac{b c r \tau f(x)}{a n^{*}}\right]\left[\frac{b c}{a}+\frac{r \tau}{n^{*}}-\frac{b c r \tau x}{a n^{*}}\right] \\
& \times \exp \left\{\left(2-\frac{x+f(x)}{n^{*}}\right) r \tau\right\} \\
= & k^{2} \exp \left\{\left(2-\frac{x+f(x)}{n^{*}}\right) r \tau\right\}\left[\left(\frac{b c}{a}+\frac{r \tau}{n^{*}}\right)^{2}\right. \\
& \left.-\left(\frac{b c}{a}+\frac{r \tau}{n^{*}}\right) \frac{b c r \tau}{a n^{*}}(x+f(x))+\left(\frac{b c r \tau}{a n^{*}}\right)^{2} x f(x)\right] \\
\leq & k^{2} \exp \left[\left(2-\frac{x+f(x)}{n^{*}}\right) r \tau\right]\left(\frac{b c}{a}+\frac{r \tau}{n^{*}}-\frac{b c r \tau(x+f(x))}{2 a n^{*}}\right)^{2} \\
= & \left(f^{\prime}\left(\frac{x+f(x)}{2}\right)\right)^{2} .
\end{aligned}
$$

By direct calculation we have

$$
f^{\prime \prime}(x)=k\left[\frac{2 b c r \tau}{a n^{*}}+\left(\frac{r \tau}{n^{*}}\right)^{2}\left(1-\frac{b c x}{a}\right)\right] \exp \left[\left(1-\frac{x}{n^{*}}\right) r \tau\right] .
$$

It is evident that $f^{\prime \prime}(x)>0$ if $0 \leq x \leq a /(b c)$. Thus $f^{\prime}(x)$ is strictly increasing on $[0, a /(b c)]$. Since $f^{\prime}(x)<0$ on $[0, a /(b c)]$, we know that $\left(f^{\prime}(x)\right)^{2}$ is strictly decreasing when $x \in[0, a /(b c)]$ and that

$$
g^{\prime}(x)>0 \text { for } x \in[0, a /(b c)] .
$$

Set $h(x)=(x+f(x)) / 2, x \in[0, a /(b c)]$ and assume that the function $h(x)$ is minimised $x_{0}$. Notice that $f([0, a /(b c)]) \subset[0, a /(b c)]$. We have $0 \leq h(x) \leq a /(b c)$ for $x \in[0, a /(b c)]$. It follows from (19) and the monotonicity of $\left(f^{\prime}(x)\right)^{2}$ that

$$
g^{\prime}(x) \leq\left(f^{\prime}\left(h\left(x_{0}\right)\right)\right)^{2} \quad \text { for } x \in[0, a /(b c)] .
$$

Since $h(0)=f(0) / 2=k e^{r \tau} / 2<a /(2 b c)=h(a /(b c))$ by Lemma 1 , we see that $x_{0}<a /(b c)$. If $x_{0}=0$, then $h^{\prime}(0+) \geq 0$. It follows that $f^{\prime}(0+) \geq-1$. If $x_{0} \in(0, a /(b c))$, then $h^{\prime}\left(x_{0}\right)=0$ and therefore $f^{\prime}\left(x_{0}\right)=-1$. Observe that condition (7) implies

$$
f^{\prime}\left(n^{*}\right)=-k\left(\frac{b c}{a}+\frac{r \tau}{n^{*}}-\frac{b c r \tau}{a}\right)=-\left(r \tau+\frac{k b c}{a}\right)>-1,
$$


in which $1 / n^{*}=b c / a+1 / k$ is used. We further note that $f^{\prime}(x)$ is strictly increasing. Thus we must have $x_{0}<n^{*}$.

Remember that $f(x)$ is strictly decreasing on $[0, a /(b c)]$. We have $f\left(x_{0}\right)>$ $f\left(n^{*}\right)=n^{*}>x_{0}$. As a consequence, $h\left(x_{0}\right)>x_{0}$, and therefore

$$
f^{\prime}\left(h\left(x_{0}\right)\right)>f^{\prime}\left(x_{0}\right) \geq-1 .
$$

Since $f^{\prime}(x)<0$ for $x \in[0, a /(b c)]$, (22) leads to $\left|f^{\prime}\left(h\left(x_{0}\right)\right)\right|<1$. It follows from (20) and (21) that $f^{2}$ is contracting on $[0, a /(b c)]$, and therefore, there exists a unique fixed point on this interval. The proof is completed.

Proof OF TheOREM 1. By Lemmas 1, 2 and 3 we have

$$
0<L_{0} \leq L_{i}<n(t)<M_{i} \leq M_{0}<a /(b c)
$$

if $t>t_{i}, i=1,2, \ldots$ It follows that $0<l \leq m<a /(b c)$. Since $n^{*}, l$ and $m$ are the fixed points of $f^{2}(x)$ on $[0, a /(b c)]$, Lemma 4 yields $l=m=n^{*}$. We then conclude from (23) that $\lim _{t \rightarrow+\infty} n(t)=n^{*}$. Finally, from the second equation of system (1), one can easily show that $\lim _{t \rightarrow+\infty} u(t)=u^{*}$. The proof is completed.

\section{Acknowledgements}

This work was supported by The National Science Foundation of The People's Republic of China, grants 19771067 and 19871067.

\section{References}

[1] M. A. Aizerman and F. R. Gantmacher, Absolute stability of regulator systems, (translated from Russian) (Holden Day, San Francisco, 1964).

[2] K. Gopalsamy and R. A. Ahlip, "Time delays in $n$-species competition-I; Global stability in constant environments", Bull. Austral. Math. Soc. 27 (1983) 427-441.

[3] K. Gopalsamy and P. X. Weng, "Feedback regulation of logistic growth", Inter. J. Math. Math. Sci. 16 (1993) 177-192.

[4] W. Wang and Z. Ma, "Global attractivity of a population model with feedback regulation", (reprint). 